\section{CPS-089 RETREATMENT OF PATIENTS WITH HEPATITIS C VIRUS INFECTION AFTER VIROLOGICAL FAILURE TO DIRECT-ACTING ANTIVIRALS}

${ }^{1} \mathrm{C}$ Burgui, ${ }^{2} \mathrm{R}$ Juanbeltz, ${ }^{2} \mathrm{~J}$ Castilla, ${ }^{3} \mathrm{~B}$ Larrayoz ${ }^{*},{ }^{3} \mathrm{M}$ Sarobe, ${ }^{4} \mathrm{~A}$ Perez, ${ }^{5} \mathrm{~A}$ Aguinaga, ${ }^{6} \mathrm{Jm}$ Zozaya, ${ }^{7} \mathrm{M}$ Gracia-Ruiz de Alda, ${ }^{3} \mathrm{R}$ San Miguel. ${ }^{1}$ Ciber Epidemiologia y Salud Publica Ciberesp, Hospital Pharmacy, Pamplona, Spain; ${ }^{2}$ Instituto de Salud Publica de NavarraIdisna-Ciber Epidemiología y Salud Pública Ciberesp, Transmissible Disease and Vaccination, Pamplona, Spain; ${ }^{3}$ Navarra Hospital Complex-Idisna, Hospital Pharmacy, Pamplona, Spain ${ }^{4}$ Navarra Hospital Complex-Ciber Epidemiología Y Salud Pública Ciberesp, Microbiology, Pamplona, Spain; ${ }^{5}$ Navarra Hospital Complex, Microbiology, Pamplona, Spain; ${ }^{6}$ Navarra Hospital Complex-Idisna, Gastroenterology and Hepatology, Pamplona, Spain; 'Navarra Hospital Complex, Infectious Disease, Pamplona, Spain

\subsection{6/ejhpharm-2019-eahpconf.238}

Background New oral interferon-free direct-acting antivirals (DAAs) have demonstrated high effectiveness treating chronic hepatitis C. However, a few patients still do not achieve sustained virological response (SVR).

Purpose To describe those patients treated with new interferon-free DAAs for chronic hepatitis C, who had virological failure (VF), and their retreatment outcomes.

Material and methods A retrospective observational study for patients with VF to DAAs who were retreated in a reference hospital from 2015 to September 2018. Variables analysed: sex, age, genotype, HIV co-infection, METAVIR score (F0F4), DAA treatment, retreatment therapy, presence of resistance-associated substitutions (RASs) and SVR 12 weeks after the end of retreatment (SVR12).

Results Twenty-four of 1356 patients treated for hepatitis C virus with interferon-free DAAs therapies had a VF (1.8\%). Seventeen were retreated (seven are pending). Median age was 51 years (36-60), 88\% male. Two patients were HIV coinfected. Genotypes: G1a $(n=6) ; \mathrm{G} 1 \mathrm{~b}(\mathrm{n}=5) ; \mathrm{G} 2(\mathrm{n}=1) ; \mathrm{G} 3$ $(n=4)$; and G4 $(n=1)$. Based on METAVIR score: F4 $(n=6)$; F3 $(n=3)$; F2 $(n=4)$; and F0-F1 $(n=4)$. Previous DAAs treatments were: ombitasvir/paritaprevir/ritonavir/ dasabuvir \pm ribavirin (RBV) $(\mathrm{n}=7)$; ledipasvir/sofosbuvir (SOF) \pm RBV $(n=3)$; daclatasvir/SOF \pm RBV $(n=2)$; velpatasvir/ SOF $(n=1)$; glecaprevir/pibrentasvir $(n=1)$; elbasvir/grazoprevir $(\mathrm{n}=1)$; SOF +RBV $(\mathrm{n}=1)$; and ombitasvir/paritaprevir/ritonavir + RBV $(n=1)$. Patients retreatment: SOF/velpatasvir/voxilaprevir $(\mathrm{n}=9) ; \quad$ elbasvir/grazoprevir/SOF $\pm \mathrm{RBV} \quad(\mathrm{n}=5)$; daclatasvir/ SOF \pm RBV $(n=1)$; velpatasvir/SOF +RBV $(n=1)$; and simeprevir/SOF +RBV $(n=1)$. Fifteen patients $(88 \%)$ were studied for RASs: four had only an available post-treatment sample and all presented a RAS related to the first DAA treatment; three had RASs at baseline and post-treatment samples; and in eight patients the RAS was only present in a post-treatment sample. SVR12 figures were available for 14 patients: 13 reached a SVR and one rebounded (three patients did not yet have analysis). Per protocol analysis, the rate of SVR was $93 \%$ for those retreatments. The patient that rebounded was G3, F4, co-infected, received at first daclatasvir/SOF +RBV during 24 weeks and was retreated with velpatasvir/ SOF + RBV during 24 weeks.

Conclusion Although only a reduced proportion of treated patients did not achieve a SVR with DAA combinations, retreatment with a new strategy reached $93 \%$ of SVR.

\section{REFERENCES AND/OR ACKNOWLEDGEMENTS}

EIPT-VHC project funded by the Spanish Ministry of Health and Carlos III Institute of Health.

No conflict of interest.

\section{CPS-090 HEALTH OUTCOMES USING DIRECT-ACTING ANTIVIRAL DRUGS FOR THE TREATMENT OF PATIENTS WITH HEPATITIS C VIRUS AND F0-F1 LIVER FIBROSIS STAGE}

M Martín López*, B Hernández Muniesa, A Ontañón Nasarre, A Pou Alonso, N Herrero Muñoz, M García Gil. Hospital Universitario de Fuenlabrada, Pharmacy, Fuenlabrada, Spain

\subsection{6/ejhpharm-2019-eahpconf.239}

Background In clinical trials, direct-acting antiviral (DAA) drugs stop hepatitis $\mathrm{C}$ virus (HCV) proliferation with efficacy rates near $100 \%$ in $\mathrm{F} 0-\mathrm{F} 1$ patients.

Purpose To describe and analyse the effectiveness and safety profiles of DAA drugs in patients with F0-F1 fibrosis stage in a university hospital.

Material and methods A retrospective, observational study. All patients with F0-F1 fibrosis stage who had initiated their treatment with DAA drugs between August 2015 and March 2018 were included. The variables collected from electronic clinical history were: demographics (age, sex), virus genotype, previous antiviral treatment, DAA agent and duration of the treatment, HIV coinfection, adverse events (AE) reported by patients and sustained viral response at week 12 (SVR12) defined as a viral load in blood less than $15 \mathrm{UI} / \mathrm{ml} 12$ weeks after having completed the treatment.

Results One-hundred and sixty-five patients were included: the median age was 53.5 years (48.5-60.5), 70 (42.4\%) were males and 16 (9.7\%) HIV-coinfected.

The most common virus was genotype $1 \mathrm{HCV}$, with 135 $(81.8 \%)$ infected patients. Sixteen $(9.7 \%)$ were infected with genotype 4, $10(6.1 \%)$ with genotype 3 and four (2.4\%) with genotype $2 \mathrm{HCV}$.

ne-hundred and forty-eight patients $(89.7 \%)$ were treatment-naive. Of the total, $48(29.1 \%)$ were treated with ledipasvir/sofosbuvir, 40 (24.2\%) with elbasvir/grazoprevir and 32 (19.4\%) with ombitasvir/paritaprevir/ritonavir/dasabuvir, 19 (11.5\%) with glecaprevir/pibrentasvir, 14 (8.5\%) with velpatasvir/sofosbuvir, seven (4.2\%) with ombitasvir/paritaprevir/ ritonavir and five (3.0\%) with other sofosbuvir-based regimens.

The effectiveness rate was 95.8\%: 158 patients achieved the SVR12. One patient $(0.6 \%)$ abandoned the treatment, and three $(1.8 \%)$ completed it but were lost to medical follow-up before determining SVR12. Three patients (1.8\%) had undetectable viral load during the treatment, but they did not achieve SVR12. Further analysis confirmed mutations of the virus genotypes, which had made them resistant to treatment.

Seventy-seven patients (46.7\%) were cured after only 8 weeks of antiviral treatment, $78(47.3 \%)$ after 12 and three $(1.8 \%)$ after 16 week treatment.

No patient left the treatment because of AE. During the treatment, $37(22.4 \%)$ felt more tired, 21 (12.7\%) had headaches, eight (4.8\%) had gastrointestinal $\mathrm{AE}$ and seven (4.2\%) experienced itching.

Conclusion Our real-world data coroborates clinical trials. The high effectiveness rates and good safety profiles of DAA permits the treatment of F0-F1 patients, which may help to eradicate $\mathrm{HCV}$ infection, a public health problem.

\section{REFERENCES AND/OR ACKNOWLEDGEMENTS}

No conflict of interest. 